\title{
Reflexiones sobre la Gobernanza Europea (*)
}

\author{
José Luis Rivero Ysern \\ Catedrático de Derecho Administrativo \\ Universidad Hispalense

\section{INTRODUCGIÓN: ALCANGE DEL CONGEPTO GOBERNANZA EUROPEA}

El Diccionario de la Real Academia Española de la Lengua define el concepto Gobernanza como el "Arte o manera de gobernar que se propone como objetivo el logro de un desarrollo económico social e institucional duradero, promoviendo un sano equilibrio entre el Estado y la Sociedad civil y el mercado de la Economía".

El concepto podría convencionalmente coincidir con el de " buen gobierno " tan al uso en las ciencias sociales y en nuestra historia. D. Quijote hablaba ya a Sancho sobre este arte de tomar buenas decisiones y le advertía del riesgo de las malas pragmáticas y de las leyes que no se cumplen.

Es positivo y aleccionador que una organización internacional como la Unión Europea se plantee a estas alturas que su manera de gobernar puede que ni haya sido ni sea la adecuada. Puede criticarse un cierto oportunismo en plantear esta cuestión en la antesala de un proceso constituyente o re-constituyente pero que duda cabe que "mas vale tarde que nunca", que recuerda nuestro refranero.

Lo cierto es que, según reconoce el "Libro Blanco de la Comisión Europea sobre la Gobernanza": "Los dirigentes políticos de toda Europa se enfrentan hoy en día a una verdadera paradoja: por una parte, los ciudadanos europeos esperan de ellos que proporcionen soluciones a los grandes proble-

\footnotetext{
* El presente trabajo recoge, sustancialmente, la intervención de su autor en las Jornadas sobre el malogrado Proyecto de Tratado por el que se instituye una Constitución para Europa, jornadas celebradas en el pasado mes de mayo en la Facultad de Derecho de la Universidad Hispalense. Con él su autor quiere adherirse al homenaje a D. Enrique Barrero González, letrado Jefe de los servicios jurídicos del Exmo Ayuntamiento de Sevilla y colaborador de nuestro Departamento de Derecho administrativo en el año de su jubilación.
} 
mas de nuestras sociedades. Por otra parte, estos mismos ciudadanos tienen cada vez menos confianza en las Instituciones y la política, o bien, simplemente, se desentienden de ello" El Parlamento europeo, por su parte, en la Resolución emitida sobre el Libro Blanco de la Comisión, con fecha 9 de noviembre de 2001, afirmó que una reforma de la "Gobernanza" europea "ha de estar basada en un análisis crítico fundamental del déficit democrático, plasmado en un informe sobre la situación de los principios de legitimación, parlamentarismo, publicidad, control y participación”.

Estas palabras del Parlamento centran realmente el alcance del proceso de Gobernanza.

Estamos ante una reflexión de la Unión Europea, propuesta por su propio ejecutivo, sobre la propia legitimidad de funcionamiento de las Instituciones Europeas. La reflexión no se reduce a cuestiones relativas a una gestión más o menos eficaz y participativa (que también); la reflexión cuestiona la adecuación de las Instituciones europeas al principio de democracia representativa, base de la Construcción europea.

El punto de partida para esta revisión del Buen Gobierno de la Unión fue una Encuesta pública cursada a las distintas instituciones de la Unión, al Comité de las Regiones a las autoridades locales y regionales y a la sociedad civil sobre el Libro Blanco de la Gobernanza Europea aprobado por la Comisión el 25 de julio de 2001.

\section{LOS RESULTADOS DE LA ENGUESTA}

El 31 de marzo 2002 finalizó la referida consulta cuyas aportaciones mas relevantes a nuestro juicio serían las siguientes:

-- Un apoyo amplio a la definición que se realiza en el Libro Blanco de los principios en que se fundamenta la gobernanza de la UE, es decir, apertura, participación, responsabilidad, eficacia y coherencia, como principios constitutivos de una buena gobernanza. En varias contribuciones se añadieron otros principios, como la legitimidad democrática y la subsidiariedad.

-- Aplaudir al Libro Blanco por haber abordado el tema de la baja participación e implicación ciudadana en la UE, aunque se considera que no se abordan suficientemente las causas de las dificultades y que las soluciones propuestas son inadecuadas. 
Se muestra preocupación por el hecho de que el análisis y el planteamiento del Libro Blanco no hagan sino reflejar una visión definida por el propio interés institucional de la Comisión y el papel del ejecutivo.

En algunas respuestas se consideraba limitado el alcance de la agenda de gobernanza propuesta en el Libro Blanco, centrado principalmente en la efectividad y la eficacia del sistema de toma de decisiones. Una parte significativa de las contribuciones hacían extensivo el objeto del debate a la propia legitimidad democrática en relación con las instituciones comunitarias y algunas relativas al planteamiento del Libro Blanco.

-- Se insiste en lo ineludible de efectuar consultas y de la participación en la elaboración de las políticas de la UE, por parte de la sociedad civil organizada y de los agentes regionales y locales en la búsqueda de una mayor participación en la elaboración de las políticas de la UE.

-- Es unánime la idea de que la participación exige como requisito previo la información: en muchas contribuciones se ha argumentado que, para una mayor participación de los ciudadanos, se necesita que las instituciones de la UE transmitan más y mejor información y que se tenga más y mejor información sobre ellas. Por lo general, se acoge favorablemente el reglamento sobre el acceso a los documentos (CE 2001/1049), pero el acceso a los documentos, por sí solo, no ofrece suficiente información sobre el proceso de toma de decisiones de la UE. Se necesita un gran esfuerzo para informar a los ciudadanos, lo que contribuiría a luchar contra el distanciamiento y a mejorar la participación.

- La consulta y la implicación de la sociedad civil no debería socavar los sistemas representativos. En un gran número de respuestas, se entiende claramente y se subraya que la consulta y la implicación de la sociedad civil no deberían socavar los sistemas representativos.

Es más, una mayoría de los diferentes interesados es bastante escéptica con la afirmación de que la mayor participación suponga y esté condicionada a una mayor responsabilidad de los actores de la sociedad civil. Existe una falta de acuerdo sobre los posibles requisitos de las estructuras internas de las organizaciones de la sociedad civil. Los actores de la sociedad civil consideran que la Comisión debería garantizar la representatividad teniendo en cuenta todos los puntos de vista. 


\section{-- Necesidad de establecer contacto con las autoridades públicas infrana- cionales}

Los comentarios a las propuestas de una mayor participación de los niveles regional y local en la elaboración de las políticas (diálogo con las autori dades regionales y locales) y en la aplicación de las políticas (contratos tripartitos por objetivos) procedían principalmente de las partes afectadas, lo que pone de manifiesto que existe un interés, pero se pide generalmente una clarificación de las ideas de la Comisión. Las respuestas revelan la existencia de una división entre la reacción crítica o escéptica de los gobiernos nacionales (el Tratado solamente regula la relación entre las autoridades nacionales y las instituciones europeas, por lo que se considera que la participación de autoridades infranacionales en la toma de decisiones de la UE es una intrusión en la organización interna de los Estados miembros) y el Parlamento Europeo, por una parte, y el positivo interés mostrado por la mayor parte de las regiones y los municipios, por otra. En varias contribuciones se pide un diálogo permanente y estructurado directo con la UE, y no a través de las asociaciones europeas y nacionales.

.- Los agentes regionales y locales piden una «subsidiariedad vertical». En una parte importante de las respuestas de las autoridades regionales y locales se menciona el concepto de «subsidiariedad vertical». Esta subsidiariedad vertical debería consagrarse necesariamente en un nuevo Tratado. Por el contrario también se expresa la preocupación de que un «estatuto especial en el Tratado» para las regiones reconocidas constitucionalmente solamente provoque una mayor desigualdad entre las regiones.

.- Un estatuto reforzado para el Comité de las Regiones en contraposición con la reforma. Se recibieron diferentes comentarios de los interesados sobre el posible papel del Comité de las Regiones. En algunas contribuciones se abogaba por un estatuto reforzado para el Comité de las Regiones, mientras que otros han abordado la necesidad de una revisión institucional completa a fin de que el Comité represente mejor a las regiones y sea más proactivo.

-- Parece necesario caminar hacia una mejora de las políticas y del proceso legislativo de la UE en la búsqueda de mejores políticas y normativas. Hay que llegar a un compromiso entre el método comunitario, la primacía institucional de un lado y la eficacia, de otro. Las respuestas apoyaron generalmente una ampliación de la gama de instrumentos políticos de la UE y un mayor uso de instrumentos no legislativos sin perjuicio de la necesidad de reconciliar estas dos consideraciones. Por una parte, existe el punto de vista de 
que, en muchos casos, los modelos reguladores alternativos y los instrumentos no legislativos han demostrado ser más eficaces y efectivos que la legislación tradicional. Por otra, se considera que una mayor eficacia no puede justificar una transferencia de la competencia de toma de decisiones a partes interesadas que no están sometidas a un control democrático.

-- Mejor preparación de la normativa. El compromiso de publicar directrices sobre el recurso a expertos por parte de la Comisión ha recibido una respuesta favorable. En las contribuciones se ha abogado por la necesidad de que las propuestas de políticas se fundamenten en criterios científicos sólidos, y se ha subrayado la importancia de un asesoramiento independiente e identificable. Asimismo, se ha aceptado en las respuestas que una evaluación ex-ante del impacto contribuirá al equilibrio y la calidad de la toma de decisiones.

Por lo que respecta a la elección del instrumento político, las opiniones están divididas. Mientras que una parte de las opiniones, en aras de la claridad, se decantan por normas fijas sobre los ámbitos políticos y los instrumentos relacionados con ellos, otras opiniones, por razones de flexibilidad, prefieren que la elección del instrumento político se decida caso por caso.

También se postulan, con apoyo desigual, los Instrumentos reguladores alternativos (corregulación, autorregulación y método abierto de coordinación).Una gran parte de las respuestas del público, especialmente de los agentes socioeconómicos, son favorables a la evaluación de todos los instrumentos políticos, incluidas las nuevas alternativas, en condiciones de igualdad. Los agentes institucionales (en particular el Parlamento Europeo) se muestran más reticentes y consideran que se precisa un nuevo examen.

-- Comitología: cuestiones relativas a una posible reforma.- Las respuestas del mundo académico e institucional han mostrado su oposición a la propuesta de la Comisión de abolir los procedimientos de los comités reguladores y de gestión y solamente mantener los procedimientos consultivos. Se considera que el Libro Blanco no ofrece alternativas al control por el Consejo de las competencias ejecutivas de la Comisión, y que una modificación de las competencias de aplicación de la UE reduciría la legitimidad democrática de las decisiones tomadas. También se ha afirmado que la abolición de los comités reguladores y de gestión supondría la sustitución de los procedimientos de búsqueda de consenso por unas competencias de la Comisión no sometidas a control. Asimismo, se ha señalado que la reforma de la comitología va más allá del marco de la gobernanza, lo que requiere un cambio en el Tratado (artículo 202 del Tratado CE). 
-- Agencias reguladoras: se expresan reservas sobre una toma de decisiones dispersas.- En la mayor parte de las respuestas al Libro Blanco se han expresado reservas sobre la creación de agencias reguladoras a nivel de la CE. En algunas contribuciones se ha señalado el hecho de que el Tratado solamente otorga competencias de administración a la $\mathrm{CE}$ en unas pocas áreas en las que se considera que la administración comunitaria es el único medio eficaz para efectuar la aplicación. Se argumenta que todavía no se ha demostrado la necesidad de agencias reguladoras a nivel de la CE. En otras respuestas se han planteado cuestiones relativas a la transparencia y el control democrático, mientras que en algunas se ha puesto en duda el valor añadido de crear nuevos estratos de participantes y se ha señalado el riesgo de que el proceso de toma de decisiones sea aún menos comprensible para los ciudadanos.

-- El método comunitario: Se apoya ampliamente la adhesión del Libro Blanco al método comunitario. Se han recibido muchas respuestas a la propuesta de centrar el papel legislativo del Parlamento Europeo y del Consejo en los principios esenciales, dejando los detalles técnicos y la aplicación a la Comisión, pero también se han planteado muchos interrogantes. Se propone que este papel ampliado previsto para la Comisión cubra muchas opciones políticas situadas por debajo del nivel de los «principios esenciales», pero que pueden seguir siendo políticas en gran medida.

-- Gobernanza de la UE: la legitimidad democrática de las instituciones de la UE. Se reconoce ampliamente que no debería considerarse que los principios de la buena gobernanza equivalen a un gobierno democrático, ya que una mejor gobernanza no puede ser la respuesta a un problema de déficit democrático. En una parte de las respuestas del público se afirma que el tema clave es la legitimidad democrática, que presupone que las decisiones se toman a través de una deliberación representativa. Por lo general, se reconoce que la petición del Libro Blanco de incluir más actores en el proceso político, si bien es necesaria, no conduce por sí misma a una mayor legitimidad democrática de las políticas o las instituciones. A este respecto, se acepta que los mecanismos de la gobernanza que tratan de reforzar la eficacia y la eficiencia del sistema de toma de decisiones y garantizar una mejor participación de más actores harán que las instituciones sean más abiertas, lo que desembocará en una mayor capacidad de respuesta y responsabilidad de las instituciones.Aunque ello podrá implicar que, seguramente, se tendrá que crear un procedimiento específico para la protección de los derechos fundamentales, y, además, se tendrán que establecer criterios en relación con la admisibilidad de las demandas, con el fin de que el Tribunal de Justicia no se bloquee como consecuencia de un alud de casos. Y quizás también se tenga que reestructurar el 
propio Tribunal, para crear una sala especializada en la protección de derechos fundamentales.

\section{LA GOBERNANZA EUROPEA Y EL PROCESO CONSTITUYENTE}

El Libro Blanco sobre la gobernanza europea había presentado propuestas clave de cambio en cuatro grandes líneas de actuación: «mayor participación», «mejores políticas, mejores normativas y mejores resultados», «la contribución de la Unión Europea a la gobernanza mundial» y «centrar las políticas y las instituciones»

\section{La primera de estas líneas: la mayor participación}

En la primera de estas líneas, la mayor participación, el Libro Blanco de la Comisión preveía una participación en sentido ascendente a través de varios procesos y actores. En una primera línea de actuación habría que hacer más abierto el método de trabajo de la Unión potenciando la labor del Parlamento Europeo y el Consejo que deberían transmitir más rápidamente la información sobre todas las fases del proceso de toma de decisiones. Hay que dar a la Unión Europea la capacidad para elaborar y difundir mensajes adaptados y concebidos en función del público al que se destinan sobre los diferentes temas de información prioritarios.

¿Como se ha llevado esto a efecto?: Poniendo en marcha un importante servicio informativo a través de Internet (Red Solvit; sitio Webb "Tu voz en Europa" que forma parte de la iniciativa de Elaboración interactiva de las políticas EIP. Base de Datos PrelexPotal Eurlex.

Se han logrado grandes mejoras en la transparencia del trabajo de las instituciones. Ha entrado en vigor el Reglamento del Parlamento Europeo y del Consejo relativo al acceso del público a los documentos del Parlamento Europeo, del Consejo y de la Comisión. Asimismo, la Comisión ha modificado su reglamento interno. Desde enero de 2002 pueden consultarse en internet las actas de las reuniones de la Comisión. Desde junio puede consultarse en línea un registro público de los documentos de la Comisión y se ha distribuido ampliamente una guía del ciudadano sobre el acceso a estos documentos con arreglo a las disposiciones del nuevo reglamento.

El Parlamento Europeo ha aprobado una serie de medidas internas (modificación de su reglamento interno y de las Obligaciones de los funcionarios y agentes del Parlamento Europeo) relativas a la aplicación del Reglamento men- 
cionado, y en junio de 2002 permitió el acceso público a su registro oficial. El registro del Consejo, que puede consultarse en línea, contiene referencias a los documentos del Consejo producidos desde el año 1999 inclusive, y muestra qué documentos se han puesto a disposición del público y en qué casos puede mostrarse directamente su contenido a partir de la base de datos o puede obtenerse utilizando el servicio de acceso a los documentos del Consejo.

La Comisión ha pedido al Comité de las Regiones que desempeñe un papel más proactivo en el examen de las políticas, por ejemplo mediante la preparación de informes exploratorios previos a las propuestas de la Comisión, para organizar el intercambio de las mejores prácticas sobre la manera de conseguir la participación de las autoridades locales y regionales en la fase preparatoria de la toma de decisiones europeas a nivel nacional. La Comisión reconoció también en su Libro Blanco sobre la gobernanza europea la necesidad de reforzar las relaciones con las autoridades regionales y locales a través de sus asociaciones nacionales y europeas. Hay que llegar hasta los ciudadanos a través de la democracia regional y local.

El Libro Blanco pedía una apertura y una consulta general de los actores de la sociedad civil en la elaboración de las políticas comunitarias. La Comisión ha adoptado principios generales y normas mínimas de consulta de partes interesadas no institucionales para conocer con exactitud quién debe ponerse en contacto con quién cuando se está elaborando una nueva política, con el objetivo de que todas las partes afectadas por la propuesta puedan implicarse más, y en condiciones de mayor igualdad, en el proceso. Es necesaria la participación de la sociedad civil. Una consulta más eficaz y más abierta en la ela boración de las políticas comunitarias.

La Comisión sigue estudiando la posibilidad de crear acuerdos de asociación más amplios con una serie de sectores organizados de la sociedad civil. El Parlamento Europeo tuvo especial cuidado en no conceder a las organizaciones de la sociedad civil un papel que, en su totalidad o en parte, coincidiera con el de quienes poseen responsabilidades políticas y han sido elegidos por sufragio universal. En algunos sectores de la sociedad civil se expresó el temor de que se creara un régimen de facto de asociaciones privilegiada.

El Comité Económico y Social Europeo ha dado una acogida favorable al Libro Blanco. En su dictamen sobre el Libro Blanco de marzo de 2002, el Comité señalaba la necesidad de garantizar el respeto de la subsidiariedad horizontal y vertical y de definir criterios que establezcan la representatividad de las organizaciones de la sociedad civil. 
Segunda línea de actuación: Mejores políticas, mejores normativas y mejores resultados

Se han conseguido rápidamente importantes progresos por lo que respecta a los compromisos del Libro Blanco en materia de mejores políticas, normativas y resultados.

-- mejores políticas. La Comisión ha emprendido iniciativas destinadas a introducir mejoras en el ciclo de elaboración de las políticas: concepción de las políticas, elección de los instrumentos y aplicación y cumplimiento de las normas. La Comisión, al emprender estas iniciativas, se ha visto alentada e inspirada por el gran volumen de trabajo preparatorio efectuado por los Estados miembros y las organizaciones internacionales, así como por la respuesta generalmente positiva de los interesados a las líneas básicas del Libro Blanco.

.- Mejores normativas. Tal como se anunció en el Libro Blanco, la Comisión propuso en junio de 2002 un amplio Plan de acción «Simplificar y mejorar el marco regulador» con el objetivo global de crear una nueva cultura legislativa común dentro de la UE.

El Plan de acción «simplificar y mejorar el marco regulador» también planteó el asunto más amplio de la elección de los instrumentos apropiados para la acción comunitaria, sobre el que la Comisión se reservó el derecho de efectuar propuestas adicionales en función del progreso de los trabajos de la Convención sobre el futuro de la Unión.

La Comisión señaló que los reglamentos y las directivas deben utilizarse de conformidad con el espíritu y la letra del Tratado CE: un reglamento solamente debería utilizarse para una acción que deba aplicarse de manera uniforme en los Estados miembros y una directiva debería respetar la definición original de la directiva tal como se establece en el Tratado y ser un instrumento por el que se crea un marco jurídico y se fijan los objetivos que deben alcanzarse.

La Comisión propuso en el Libro Blanco, y posteriormente en el Plan de acción «simplificar y mejorar el marco regulador», utilizar en mayor medida las alternativas a la legislación tradicional sin socavar las disposiciones del Tratado o las prerrogativas del legislador.

Existen varios instrumentos que pueden utilizarse en circunstancias específicas para conseguir los objetivos del Tratado y, al mismo tiempo, simplificar 
las actividades legislativas y la propia legislación, corregulación, autorregulación y método abierto de coordinación. Recordemos: en el marco de un acto legislativo, la corregulación permite poner en práctica los objetivos definidos por el legislador mediante medidas aplicadas por partes activas y reconocidas en el ámbito en cuestión. La Comisión sigue estando convencida de que puede recurrirse a la corregulación para centrar el trabajo legislativo en sus elementos esenciales y para simplificar y mejorar la aplicación, dentro de los límites establecidos por los criterios fijados en un futuro acuerdo interinstitucional sobre un mejor marco regulador. La autorregulación afecta a un gran número de prácticas, normas comunes, códigos de conducta y acuerdos voluntarios con agentes económicos, interlocutores sociales, ONG y grupos organizados que establecen bases voluntarias para regular y organizar sus actividades. A diferencia de la corregulación, la autorregulación no implica ningún acto legislativo. La Comisión ha propuesto criterios y modalidades para la utilización de estos instrumentos alternativos y de otros similares para conseguir los objetivos del Tratado, y se están negociando en la actualidad en un marco interinstitucional. La Comisión, tras proponer orientaciones sobre el papel del método abierto de coordinación en el Libro Blanco, prevé en su estrategia política anual para 2003 la realización de una evaluación estratégica sobre el funcionamiento del método en los ámbitos en los que se ha aplicado inicialmente.

La Comisión ha propuesto que, con el apoyo del Consejo y del Parlamento Europeo, se emprenda un gran esfuerzo a fin de simplificar el acervo comunitario y reducir su volumen. La Comisión propone que las instituciones definan conjuntamente un programa de simplificación de la legislación comunitaria.

\section{Tercera línea de actuación: contribución de la UE a la gobernanza mundial}

El Libro Blanco subrayó la necesidad de conseguir una reforma con éxito de la gobernanza en la UE a fin de que ésta pueda tener credibilidad al abogar por un cambio a nivel mundial;

Cuarta línea de actuación: la Unión puede contribuir a una reforma general de las instituciones multilaterales y a mejorar la cooperación

En cuanto a la cuestión de cómo la Unión puede contribuir a una reforma general de las instituciones multilaterales y a mejorar la cooperación, la Comisión está elaborando un planteamiento más global y estratégico para la Comisión y para la UE en sus relaciones con el sistema de Naciones Unidas y las instituciones de Bretton Woods. 
El Libro Blanco registró y analizó, a nivel europeo e internacional, la creciente importancia social y política de las redes, concebidas como una interacción entre individuos u organizaciones (comunidades, autoridades regionales y locales, empresas, administraciones, centros de investigación, etc.) de manera no jerárquica y en las que cada participante es responsable de una parte de los recursos necesarios para conseguir el objetivo común, siendo la comunicación electrónica el instrumento preferido. En esta línea pueden citarse la Elaboración Interactiva de las Políticas (EIP)_a fin de poder recibir reacciones espontáneas en línea.

\section{LA GOBERNANZA EN LA CONSTITUCIÓN EUROPEA}

Varios son los preceptos que deben examinarse en el Proyecto de Constitución europea en relación a la gobernanza europea; en síntesis estos preceptos y su respectiva temática son: Parte Primera Título II. Artc.- I. 10. d. Derechos fundamentales. Ciudadanía Formular peticiones al Parlamento Recurrir al D. Del Pueblo.- Parte Primera Título VI Artcs. I.45 a 52 especialmente artc. 50. De la vida democrática de la Unión.

Parte Segunda Título V Artcs. 99 - 104 especialmente artículos 101 y 102. Ciudadanía Derecho a una buena administración.

A la vista de estos preceptos y de estos antecedentes puede formularse una pregunta clave: ¿la recogida de todos estos principios y medidas en el Proyecto de Constitución: ¿permite augurar una administración mas transparente mas eficaz, mas participativa, mas inteligible, mas cercana para el ciudadano?

No lo sé. Si se examina la opinión de los distintos sectores que han participado en el proceso de encuesta quizá faltan soluciones y propuestas de utilidad. Pensemos que es un primer paso. No olvidemos que a andar se aprende andando. Pensemos que se nos abren caminos y soluciones que debían haber estado en marcha hace muchos años pero reconozcamos también que nuestro nivel de descontento y protesta solo hoy ha sido lo suficientemente claro para que la Unión Europea reflexionara e iniciara ¿quizá? un cambio.

En cualquier caso creo que para nuestro Derecho el Proyecto de Constitución constituye, en materia de transparencia, en materia de gobernanza, un avance. Tengamos muy presente que todos los principios y derechos que hoy se formulan en este Proyecto de Texto Constitutivo en nuestro Derecho y hasta la fecha no han sido sancionados ni recogidos por nuestra Constitución de 1978 salvo para establecer una reserva de ley al respecto en algunos casos 
(artc. $105 \mathrm{CE}$ ); y que solo a nivel de ley ordinaria hemos formulado unos principios y garantías en el procedimiento administrativo (Artículo 35 de la ley $30 /$ 92) o unos principios de la organización funcionamiento y relaciones de la Administración con los ciudadanos (Artículos 3 y 4 de la Ley 6/1997 de 14 de Abril LOFAGE).

En cualquier caso lo que el Proyecto incorpora es un paso importante en el buen camino. Ese es el camino, reconocer y formular y regular esos derechos que constituyen el buen hacer de la Administrativo, la buena administración. Recogerlos, como hace el Proyecto de Tratado (cierto que parte de ellos pero en cualquier caso los mas importantes), como derechos fundamentales, es de enorme importancia.

Termino: el profesor Carrillo Salcedo contaba una anécdota llamativa acerca de la confesión de unos prestigiosos especialistas en Derecho internacional público y Derecho de la Unión europea a otros especialistas, sobre su "incomprensión" del Tratado de Maastrich.

A quien les habla la anécdota le impactó. Pensé que, realmente a estos prestigiosos profesores ningún derecho les estaba vulnerando esta "jerga ininteligible" comunitaria (como el mismo maestro Carrillo calificó los textos comunitarios). Ellos sabían del tema sin duda mucho mas que quienes habían redactado aquel Tratado.

Pero pensé en tantas personas que a lo largo de tanto tiempo habían estado sin entender de lo que se les hablaba.

Como ha recordado Fernando Sainz Moreno, ( "El legislador racional y la predecibilidad de la conducta humana" publicado en "La proliferación legislativa un desafio para el Estado de Derecho". Seminario organizado por el Colegio Libre de Eméri tos Cívitas 2004) nuestra CE garantiza la interdicción de la arbitrariedad de los poderes públicos (artc. 9.3) y exige que los "proyectos de ley vayan acompañados de los antecedentes necesarios para pronunciarse sobre ellos".

Con ello la Constitución esta asegurando y garantizando, ni mas ni menos, la racionalidad de las normas en su sentido más amplio, esto es, que hayan sido dictadas con conocimiento suficiente de aquello que se pretende regular, con una finalidad legítima, proporcionada, adecuada a este fin, y con respeto del valor justicia. Nuestro artículo $9.3 \mathrm{CE}$ y la cláusula de interdicción de la arbitrariedad de los poderes públicos son, a mi juicio la consagración del derecho de los ciudadanos a la buena gobernanza. 
Porque es efectivamente arbitrariedad, legislar sin tener los antecedentes necesarios para pronunciarse sobre los asuntos; es arbitrariedad administrar de espaldas al ciudadano.

Decía Gracián a mediados del siglo XVII. en su "Arte de la Prudencia" (y tomo prestada la cita de Sainz Moreno), que antes de decidir hay que enterarse de los asuntos y que solo actuando racionalmente se puede pensar bien.

Cabe pensar si con todo esto no estemos, en parte, redescubriendo el sentido común aplicado a la gestión de la cosa pública pero también, en los tiempos que corren, esto sería motivo de regocijo. 